\title{
Susceptibilities to Antimicrobials and Disinfectants in Salmonella Isolates Obtained from Poultry and Swine in Thailand
}

\author{
Rungtip CHUANCHUEN ${ }^{1) *}$, Pornpen PATHANASOPHON ${ }^{2)}$, Sirintip KHEMTONG ${ }^{1)}$, Wechsiri WANNAPRASAT ${ }^{1)}$ and \\ Pawin PADUNGTOD ${ }^{3)}$
}

${ }^{1)}$ Faculty of Veterinary Science, Chulalongkorn University, Bangkok 10330, ${ }^{2}$ National Institute of Animal Health, Ministry of Agriculture and Cooperatives, Bangkok 10900 and ${ }^{3)}$ Faculty of Veterinary Medicine, Chiangmai University, Chiangmai 50100 Thailand

(Received 2 October 2007/Accepted 19 February 2008)

\begin{abstract}
Salmonella enterica isolates from poultry $(n=125)$ and swine $(n=132)$ in Thailand were investigated for antibiotic resistance, susceptibility to disinfectants (benzalkonium chloride (BKC), chlorhexidine digluconate (CHX), zinc chloride and copper sulfate) and cyclohexane tolerance. Forty-two percent were of multiple resistance to antibiotics. The minimum inhibitory concentrations (MICs) of all antibiotics against isolates from swine were higher than that against the isolates from poultry. There were generally few variations in MICs to all disinfectants, indicating that the isolates had either no or only a limited degree of developed resistance to the disinfectants tested. Only 5 isolates $(1.9 \%)$ were tolerant to cyclohexane. The proton-dependent efflux systems did not play a major role in the reduced susceptibility to BKC and $\mathrm{CHX}$, since susceptibility was not restored when an efflux inhibitor, carbonyl cyanide $m$-chlorophenylhydrazone (CCCP) was present. Successive exposure to subinhibitory concentrations of BKC and CHX generated mutants resistant to BKC and $\mathrm{CHX}$. A spontaneous BKC-resistant derivative expressed cross-resistance to antibiotics, chloramphenicol and erythromycin. The mechanism responsible for cross-resistance between BKC and antibiotics was not driven by the proton motif force (PMF). KEY WORDS: benzalkonium chloride, multiple antibiotic resistance, Salmonella enterica, proton-dependent efflux system.
\end{abstract}

In food animal production, antibiotics are widely administered for therapy and the prophylaxis of bacterial infections and growth promoters, while disinfectants are used extensively for disinfection. Cationic agents such as benzalkonium chloride (BKC) and chlorhexidine ( $\mathrm{CHX}$ ) have been formulated in farm disinfectants [3]. Heavy metals i.e. copper sulfate $\left(\mathrm{CuSO}_{4}\right)$ and zinc chloride $\left(\mathrm{ZnCl}_{2}\right)$ are used as the growth-promoting feed supplements [1]. As is the case for antibiotics, bacteria may develop resistance to disinfectants and heavy metals [18] and concern that the inappropriate use of these substances may select for antibiotic resistance has been increasing.

The mechanisms of antibiotic resistance have been extensively studied, however, reduced susceptibility to biocide have been comparatively poorly elucidated. One of the mechanisms underlying insusceptibility to biocide is the presence of an active efflux pump. Although a traditional efflux pump extrudes a single substrate, multidrug efflux systems export several structurally unrelated compounds and, therefore, confer cross-resistance between antibiotics and disinfectants. Whereas the proton-dependent efflux pumps i.e. those in the major facilitator (MF) superfamily, the resistance-nodulation-division (RND) family and the small multidrug resistance (SMR) family have been commonly found in Gram-negative bacteria, the RND type efflux pumps are predominant [21]. In Salmonella, the RND family AcrAB-TolC system has been shown to confer resistance to multiple antibiotics and disinfectants resulting in multiple antibiotic resistance (MAR) phenotypes [16,

\footnotetext{
* Correspondence to: Chuanchuen, R., Faculty of Veterinary Science, Chulalongkorn University, Bangkok 10330 Thailand. e-mail: rchuanchuen@yahoo.com
}

25]. Such MAR phenotype has been shown to be associated with cyclohexane tolerance [22]. However, little is known about overall role of efflux systems using proton motif force (PMF) in reduced susceptibility to biocide in large number of Salmonella from farm sector.

Swine and poultry are the major food-producing animals in several countries and have been implicated as sources of antimicrobial-resistant Salmonella. Currently, data on susceptibility to antimicrobial agents and resistance mechanisms for Salmonella isolates in developing countries including Thailand is still limited. Susceptibility to disinfectants has been less reported and very little is known about susceptibility to heavy metals used as feed additives. The aims of the present study were to i) further investigate the prevalence of antibiotic resistance in $S$. enterica in Thailand ii) examine the susceptibility to selected disinfectants (i.e. BKC and CHX) and heavy metals used as feed supplements (i.e. copper sulfate and zinc chloride) and iii) monitor the involvement of efflux pumps driven by PMF in reduced susceptibility to BKC and $\mathrm{CHX}$.

\section{MATERIALS AND METHODS}

Bacterial strains, media and growth conditions: The $S$. enterica strains $(n=257)$ representing 44 serotypes from poultry $(n=125)$ and swine $(n=132)$ were randomly selected from the collection at National Institute of Animal Health, Bangkok, Thailand (Table 1). All the strains were isolated from samples (feces, rectal swabs, drinking water and feed) collected from healthy poultry and swine during 20032005. Only one colony of each serotype was collected from each positive sample. All isolates were stored in $20 \%$ glyc- 
Table 1. Salmonella serotypes used in this study $(n=257)$

\begin{tabular}{|c|c|c|}
\hline \multirow[t]{2}{*}{ Serotype (n) } & \multicolumn{2}{|c|}{ Number of isolates ${ }^{\text {a) }}$} \\
\hline & Poultry & Swine \\
\hline S.Albany (5) & 4 & 1 \\
\hline S.Altona (3) & 0 & 3 \\
\hline S.Agona (3) & 3 & 0 \\
\hline S.Amsterdam (11) & 11 & 0 \\
\hline S.Anatum (9) & 0 & 9 \\
\hline S. Bovismorbifican (5) & 1 & 4 \\
\hline S.Bsilla (18) & 0 & 18 \\
\hline$S$.Bareilly & 1 & 0 \\
\hline S.Biafra & 0 & 1 \\
\hline S.Blockley & 1 & 0 \\
\hline S.Corvallis (11) & 5 & 6 \\
\hline S.Djugo & 0 & 1 \\
\hline S.Enteritidis (34) & 31 & 3 \\
\hline$S$.Emek (5) & 5 & 0 \\
\hline S.Eppendorf & 1 & 0 \\
\hline S.Give (5) & 4 & 1 \\
\hline S.Hvittingfoss (3) & 3 & 0 \\
\hline S.Infantis & 1 & 0 \\
\hline S.Kedougou (10) & 1 & 9 \\
\hline S.Kingston & 0 & 1 \\
\hline S.Kentucky (3) & 3 & 0 \\
\hline S.Lexington & 1 & 0 \\
\hline S.Madjorio (2) & 2 & 0 \\
\hline S.Mbandaka & 1 & 0 \\
\hline S.Muenster & 1 & 0 \\
\hline S.Orion (3) & 1 & 2 \\
\hline S.Panama & 0 & 1 \\
\hline S.Paratyphi B & 1 & 0 \\
\hline S.Paratyphi B2 & 1 & 0 \\
\hline S.Poona (2) & 2 & 0 \\
\hline$S$. Rissen (14) & 1 & 13 \\
\hline S.Stanley (30) & 5 & 25 \\
\hline Salmonella subspecies I (12) & 4 & 8 \\
\hline S.Sainpaul & 1 & 1 \\
\hline S.Schwarzengrund & 1 & 0 \\
\hline S.Senftenberg (7) & 4 & 3 \\
\hline S.Singpore & 1 & 0 \\
\hline S.Suberu & 1 & 0 \\
\hline S. Thyphimurium (20) & 7 & 13 \\
\hline$S$.Thomson & 1 & 0 \\
\hline S.Virginia & 0 & 1 \\
\hline S.Virchow (4) & 3 & 1 \\
\hline S. Worthington (4) & 2 & 2 \\
\hline$S$. Weltevreden (14) & 9 & 5 \\
\hline Total & 125 & 132 \\
\hline
\end{tabular}

a) Total number of isolates is indicated if more than one. erol at $-80^{\circ} \mathrm{C}$. Bacteria were grown on Luria-Bertani (LB) media (Difco, MI, U.S.A.) or Muller Hinton Agar (MHA; Difco) at $37^{\circ} \mathrm{C}$.

Antimicrobial susceptibility testing: The minimum inhibitory concentration (MIC) were determined by a two-fold agar dilution technique according to the Clinical and Laboratory Standards Institute (CLSI, formerly NCCLS) [19]. Ampicillin (AMP), chloramphenicol (CHP), gentamicin (GEN), ciprofloxacin (CIP), tetracycline (TET), trimethoprim (TRI), sulphamethozaxole (SUL), BKC and CHX were purchased from Sigma (Detroit, U.S.A.). $\mathrm{CuSO}_{4}$ and $\mathrm{ZnCl}_{2}$ were obtained from UNILAB (Australia). $\mathrm{CuSO}_{4}$ and $\mathrm{ZnCl}_{2}$ were dissolved in distilled water, of which $\mathrm{pH}$ was adjusted to 5.5 for $\mathrm{ZnCl}_{2}$. Multidrug resistance (MDR) was defined as isolates being resistant to 2 or more separate classes of antibiotics [13].

Activity of the active efflux system inhibitor: Susceptibility for carbonyl cyanide $m$-chlorophenylhydrazone (CCCP, Sigma) was determined in 4 representatives of Salmonella isolates and the MIC values were 200-400 $\mu \mathrm{M}$. Therefore, the MICs of BKC and CHX were determined in the presence and absence of $50 \mu \mathrm{M}$ CCCP. The isolates were grown in the presence of CCCP without disinfectants to ensure that CCCP did not have inhibitory effect. Experiments were independently repeated twice.

Cyclohexane tolerance test: Cyclohexane tolerance was determined by the method of Asako et al. [2]. This experiment was performed in 2 separate occasions. Colonies that grew in the presence of cyclohexane were deemed cyclohexane tolerant.

PCR amplification of antibiotic-resistance genes: The presence of antibiotic-resistance genes including bla $a_{\mathrm{PSE}-1}$, $b_{\text {beM }}, \operatorname{aad} A 1, \operatorname{aad} A 2, \operatorname{dfr} A 1, \operatorname{dfr} A 12$, tet $A, \operatorname{tet} B, \operatorname{cat} A$ and $c a t B$ were determined by PCR using specific primers (Table 2). All primers were manually designed from the gene sequence available at the National Center for Biotechnology for Biotechnology Information website (www.ncbi.nlm. nih.gov) and were commercially synthesized (1st BASE Pte Ltd, Singapore Science Park II, Singapore). To confirm the specificity of PCR amplifications, PCR products were gel purified using QIAQuick Gel Extraction kit (Qiagen, Hilden, Germany) and submitted for sequencing at Macrogen Inc. (Seoul, South Korea). The genes were chosen because of their distribution in Salmonella isolates from food animals (our unpublished data). Template DNA was

Table 2. Primers used in this study

\begin{tabular}{lll}
\hline Resistance genes & \multicolumn{2}{c}{ Sequence of primers } \\
\hline bla $_{\mathrm{PSE}-1}$ & 5'-GCAAGTAGGGCAGGCAATCA-3' & 5'-GAGCTAGATAGATGCTCACAA-3' \\
bla $_{\mathrm{TEM}}$ & 5'-ATCAGTTGGGTGCACGAGTG-3' & 5'-ACGCTCACCGGCTCCAGA-3' \\
aadA1 & 5'-CTCCGCAGTGGATGGCGG-3' & 5'-GATCTGCGCGCGAGGCCA-3' \\
aadA2 & 5'-CATTGAGCGCCATCTGGAAT-3' & 5'-ACATTTCHCTCATCGCCGGC3' \\
$d f r A 1$ & 5'-CAATGGCTGTTGGTTGGA-3' & 5'-CCGGCTCGATGTCTATTGT-3' \\
$d f r A 12$ & 5'-TTCGCAGACTCACTGAGGG-3' & 5'-CGGTTGAGACAAGCTCGAAT-3' \\
tet $A$ & 5'-GCTGTCGGATCGTTTCGG-3' & 5'-CATTCCGAGCATGAGTGC-3' \\
$t e t B$ & 5'-CTGTCGCGGCATCGGTCAT-3' & 5'-CAGGTAAAGCGATCCCACC-3' \\
cat $A$ & 5'-CCAGACCGTTCAGCTGGATA-3' & 5'-CATCAGCACCTTGTCGCCT-3' \\
$c a t B$ & 5'-CGGATTCAGCCTGACCACC-3' & 5'-ATACGCGGTCACCTTCCTG-3' \\
\hline
\end{tabular}


prepared by the whole cell lysate procedure [17]. PCR was performed using Eppendrof ${ }^{\circledR}$ MasterMix (Eppendrof, Hamburg, Germany) according to the manufacturer's instructions. PCR reaction conditions were an initial denaturation at $95^{\circ} \mathrm{C}$ for $5 \mathrm{~min}$, and 30 cycles of denaturation for $45 \mathrm{sec}$ at $95^{\circ} \mathrm{C}$, primer annealing for $45 \mathrm{sec}$ at $55^{\circ} \mathrm{C}$, and extension for 45 seconds at $72^{\circ} \mathrm{C}$ and a final extension for $10 \mathrm{~min}$.

Passage of strains with BKC and CHX: Step-wise exposure to gradually increasing concentrations of $\mathrm{BKC}$ was performed in 3 isolates with low MICs to BKC and a high susceptibility to antibiotics as previously described with some modifications [10]. After an overnight incubation of $37^{\circ} \mathrm{C}$, an $80-\mu l$ inoculum was used to inoculate $4 \mathrm{~m} l \mathrm{LB}$ containing $\mathrm{BKC}$ at a concentration of half the MIC. This procedure took place everyday in LB broth with increasing concentrations of BKC by a factor of 1.5 until no growth was observed [10]. At each passage, the inoculums were cultured onto LB agar without BKC. Four randomly-picked colonies from the final passage were assayed to determine MICs for BKC and antibiotics. The colonies were subcultured in non-selective LB medium for 20 consecutive days. Then, the MIC determinations were repeated. The MICs for erythromycin were additionally examined because crossresistance between $\mathrm{BKC}$ and erythromycin had been shown in previous studies [5]. The continuity of pre- and postexposed strains was confirmed by repetitive sequence based polymerase chain reaction (rep-PCR) [26]. For CHX passage, 4 isolates with low MICs for CHX and antibiotics were selected and exposure experiments were performed using the same protocol.

Data analysis: Percentage and percentile (MIC50) was used to describe the proportion of resistance isolates and MIC value respectively. Statistical tests for significant difference between proportions of resistance isolates were performed using chi-square or Fisher's exact test. Statistical tests for significant difference between MIC values was performed using the Wilcoxon rank sum test [8]. All statistical analyses was performed on STATA 9.2 (StataCorp., TX, U.S.A.).

\section{RESULTS}

Susceptibility to antibiotics and disinfectants: All Salmonella isolates were examined for their susceptibility to 6 antibiotics and 4 disinfectants. The frequency of antibiotic resistance is shown in Fig. 1. Sixty-seven percent of all isolates were resistant to antibiotics. The MIC ranges for AMP, CHP, CIP, GEN, TET and TRI were 0.5-256, 1$>256,<0.03-4,<0.25->64,<0.25->256$ and $0.25->256$ $\mu \mathrm{g} / \mathrm{ml}$, respectively. The MIC50s of these antibiotics were $4,8,0.125,0.5,32$ and $0.5 \mu \mathrm{g} / \mathrm{ml}$, respectively. Resistance to tetracycline was observed most often in Salmonella isolated from swine. Among the isolates from poultry, the highest frequencies of resistance were the frequencies of resistance to ampicillin and tetracycline. Resistance was also observed, but to a lesser extent, for chloramphenicol, gentamicin and trimethoprim. Overall, the isolates from

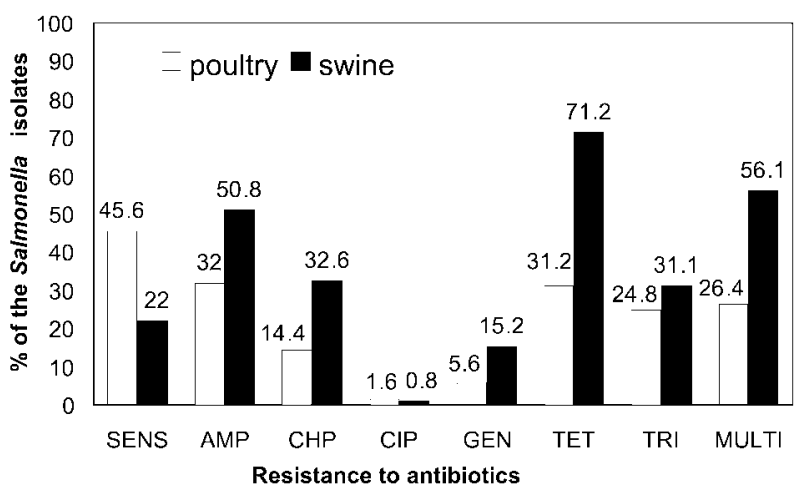

Fig. 1. Frequency of resistance to 6 antibiotics in the Salmonella isolates from poultry and swine $(n=257)$. AMP, ampicillin; CHP, chloramphenicol; CIP, ciprofloxacin, GEN, gentamicin; TET, tetracycline; TRI, trimethoprim; MULTI; multidrug resistance; SENS; sensitive to all antibiotics tested.

swine had a higher prevalence of resistance to all antibiotics examined, except ciprofloxacin and trimethoprim, than those from poultry did. Resistance patterns were also investigated (Table 3). All isolates can be grouped into 17 resistance phenotypes (16 different patterns for poultry isolates and 16 different patterns for swine isolates). Of these, 15 resistance patterns were shared by isolates from both species. The most frequent multiple resistance patterns for poultry and swine isolates were AMP-CHP-TET-TRI (5.7\%) and AMP-CHP-TET (10.4\%), respectively. The AMP-CHP-TET-TRI phenotype was the most common multiple antibiotic resistance pattern among all of the isolates $(7 \%)$.

For susceptibility to disinfectants, the MIC distributions are given in Table 4. In general, the Salmonella isolates from poultry and swine exhibited a similar susceptibility to all disinfectants and there were few deviations from general distribution in MICs to the different compounds. The median MICs (MIC50) of all disinfectant in isolates from swine were not significantly different from isolates from chicken ( $p=0.479,0.441,0.555,1.074)$.

A hundred and nineteen Salmonella isolates exhibited high MICs to BKC (128-256 $\mu \mathrm{g} / \mathrm{ml})$, while 73 Salmonella isolates showed high MICs to CHX (16-64 $\mu \mathrm{g} / \mathrm{m} l)$ (Table 3). Of all Salmonella with low susceptibility to BKC, $68.9 \%$ showed resistance to at least one antibiotic. Sixtyfive percent of Salmonella with high susceptibility to BKC were resistant to antibiotics tested. Among Salmonella isolates with high MICs to $\mathrm{CHX}, 60 \%$ demonstrated antibiotic resistance. Sixty-nine percent of those with MICs to CHX were resistant to antibiotics. Multiple antibiotic-resistant Salmonella isolates were not less susceptible to BKC or $\mathrm{CHX}$ than those non-multidrug resistant strains $(\mathrm{p}=0.478$, 0.485).

Influence of the efflux pump inhibitor: The ability of CCCP to influence MIC values was determined in 119 isolates with high MICs for BKC and 73 isolates with high MICs for CHX. The antibiotic resistance patterns of the 
Table 3. Antibiotic resistance patterns of Salmonella isolates in this study

\begin{tabular}{|c|c|c|c|c|c|c|}
\hline \multirow[t]{2}{*}{ Antibiotic resistance pattern } & \multicolumn{2}{|c|}{ Number of isolates $(\%)^{\mathrm{a})}$} & \multicolumn{2}{|c|}{ Number of isolates $(\%)^{b)}$} & \multicolumn{2}{|c|}{ Number of isolates $(\%)^{c)}$} \\
\hline & Poultry & Swine & High BKC & Low BKC & High CHX & Low CHX \\
\hline AMP & $16(13.1)$ & $2(1.5)$ & $9(7.6)$ & $9(6.5)$ & $4(5.5)$ & $14(7.6)$ \\
\hline CHP & $2(1.6)$ & $2(1.5)$ & $3(2.5)$ & $1(0.7)$ & $2(2.7)$ & $2(1.1)$ \\
\hline TET & $11(9.0)$ & $24(17.8)$ & $10(8.4)$ & $25(18.1)$ & $9(12.3)$ & $26(14.1)$ \\
\hline TRI & $6(4.9)$ & $1(0.7)$ & $2(1.7)$ & $5(3.6)$ & $2(2.7)$ & $5(2.7)$ \\
\hline AMP-CIP & $2(1.6)$ & $1(0.7)$ & 0 & $3(2.2)$ & 0 & $3(1.6)$ \\
\hline AMP-TET & $5(4.1)$ & $10(7.0)$ & $9(7.6)$ & $6(4.3)$ & $4(5.5)$ & $11(6.0)$ \\
\hline AMP-TRI & $1(0.8)$ & $2(1.5)$ & 0 & $3(2.2)$ & 0 & $3(1.6)$ \\
\hline CHP-TRI & $2(1.6)$ & $1(0.7)$ & $3(2.5)$ & 0 & 0 & $3(1.6)$ \\
\hline TET-TRI & $4(2.5)$ & $7(5.2)$ & $4(3.4)$ & $7(5.1)$ & $5(6.8)$ & $6(3.3)$ \\
\hline AMP-CHP-TET & $1(0.8)$ & $14(10.4)$ & $11(9.2)$ & $4(2.9)$ & $8(11)$ & $7(3.8)$ \\
\hline AMP-TET-TRI & $2(1.6)$ & $7(5.2)$ & $5(4.2)$ & $4(2.9)$ & $2(2.7)$ & $7(3.8)$ \\
\hline CHP-GEN-TET & 0 & $1(0.7)$ & 0 & $1(0.7)$ & 0 & $1(1.6)$ \\
\hline CHP-TET-TRI & $3(2.5)$ & 0 & $1(0.8)$ & $2(1.4)$ & 0 & $3(1.6)$ \\
\hline AMP-CHP-GEN-TET & $1(0.8)$ & $7(5.2)$ & $8(2.5)$ & 0 & $2(2.7)$ & $6(3.3)$ \\
\hline AMP-CHP-TET-TRI & $7(5.7)$ & $11(8.1)$ & $10(8.4)$ & $8(5.8)$ & $3(4.1)$ & $15(8.2)$ \\
\hline AMP-GEN-TET-TRI & $3(2.5)$ & $6(4.4)$ & $3(2.5)$ & $6(4.3)$ & $1(1.4)$ & $8(4.3)$ \\
\hline AMP-CHP-GEN-TET-TRI & $3(2.5)$ & $6(4.4)$ & $4(3.4)$ & $5(3.6)$ & $2(2.7)$ & $7(3.8)$ \\
\hline
\end{tabular}

a) Salmonella isolates from poultry $(n=122)$ and swine $(n=135)$.

b) Salmonella isolates exhibiting low $(n=119)$ and high $(n=138)$ susceptibility to BKC.

c) Salmonella isolates exhibiting low $(n=73)$ and high $(n=184)$ susceptibility to CHX.

Table 4. Susceptibility of the Salmonella enterica isolates from poultry $(n=122)$ and swine $(n=135)$ to benzalkonium chloride, chlorhexidine, zinc chloride and copper sulfate

\begin{tabular}{|c|c|c|c|c|c|c|c|c|c|c|c|c|c|c|}
\hline \multirow[t]{2}{*}{ Agent } & \multirow[t]{2}{*}{ Sources } & \multirow[t]{2}{*}{ MIC50 } & \multirow{2}{*}{$\begin{array}{c}\text { P- } \\
\text { Value }^{\text {a) }}\end{array}$} & \multicolumn{11}{|c|}{$\%$ of isolates with MICs $(\mu \mathrm{g} / \mathrm{m} l)$} \\
\hline & & & & 2 & 4 & 8 & 16 & 32 & 64 & 128 & 256 & 512 & 1024 & 2048 \\
\hline \multirow[t]{2}{*}{$\mathrm{BKC}$} & Poultry & 64 & 0.48 & & & & & 12.0 & 41.6 & 32.0 & 14.4 & & & \\
\hline & Swine & 64 & & & 0.8 & & 0.8 & 6.8 & 45.5 & 25.0 & 21.2 & & & \\
\hline \multirow[t]{2}{*}{$\mathrm{CHX}$} & Poultry & 8 & 0.44 & 1.6 & 4.0 & 68.0 & 17.6 & 8.0 & 2.4 & & & & & \\
\hline & Swine & 8 & & & 13.4 & 59.8 & 23.5 & 4.5 & 0.8 & & & & & \\
\hline \multirow[t]{2}{*}{$\mathrm{ZnCl}_{2}$} & Poultry & 1024 & 0.56 & & & & & & & & & 14.4 & 50.4 & 35.2 \\
\hline & Swine & 1024 & & & & & & & & & & 15.9 & 43.2 & 40.9 \\
\hline \multirow[t]{2}{*}{$\mathrm{CuSO}_{4}$} & Poultry & 2048 & 0.07 & & & & & & & & & 0.8 & 1.6 & 97.6 \\
\hline & Swine & 2048 & & & & & & & & & & & & 100.0 \\
\hline
\end{tabular}

a) P-value indicates the significant level of the difference of MIC50 between swine and poultry using the Wilcoxon rank-sum test.

strains tested are shown in Table 3. Most of the strains exhibiting high susceptibility to BKC (48.7\%) and CHX $(36.9 \%)$ were resistant to multiple drugs. The presence of CCCP decreased the MICs of BKC in only one $S$. Enteritidis isolate, namely SA085. The MIC reduction was up to 16 fold (from 128 to $8 \mu \mathrm{g} / \mathrm{m} l$ ). The addition of CCCP did not affect the MICs of CHX in any isolates. In control plates, all tested isolates grew in LB agar containing $50 \mu \mathrm{M} \mathrm{CCCP}$ (data not shown).

Growth in cyclohexane and the presence of antibiotic resistance genes: All Salmonella strains were investigated for their tolerance of the organic solvent cyclohexane. Cyclohexane tolerance was observed in only one isolate from poultry $(0.8 \%)$ and 4 isolates from swine $(3.0 \%)$. The MIC values for antibiotics and disinfectants of the cyclohexane-tolerant isolates are shown in Table 5. All isolates tested grew on LB agar without cyclohexane (data not shown).

All cyclohexane tolerant isolates were tested for the presence of related antibiotic-resistance genes. SA010 and
SA034 carried bla $a_{\mathrm{TEM}}, d f r A 12$ and tetA. SA070 harbored $b l a_{\mathrm{TEM}}$ and $t e t A$. None of the resistance genes tested was present in SA059 and SA105.

Spontaneous resistance mutants and their resistance phenotype: For BKC passage, only a $S$. Stanley strain, SA030 grew beyond the first passage and its growth ceased at a concentration of $32 \mu \mathrm{g} / \mathrm{m} l$. All four randomly-picked mutants obtained from the last passage had a reduced susceptibility to BKC by 8 fold (from 4 to $32 \mu \mathrm{g} / \mathrm{m} l$ ) but only one colony, namely SA065 was additionally resistant to chloramphenicol and erythromycin (Table 6). The continuity of SA030 and its BKC-resistant derivative, SA065 was confirmed by rep-PCR profiling and the electrophoresis patterns of both strains were identical (data not shown). The presence of CCCP did not restore susceptibility to chloramphenicol and erythromycin in SA065. No changes of MICs were observed after growing on non-selective LB medium for 20 consecutive days. From a cyclohexane tolerance test, SA065 was cyclochexane susceptible.

For CHX passage, only SA027, a $S$. Enteritidis strain with 
Table 5. Minimum Inhibitory Concentrations (MICs) for antibiotics and disinfectants in the cyclohexane-tolerant isolates

\begin{tabular}{|c|c|c|c|c|c|c|c|c|c|}
\hline \multirow{2}{*}{$\begin{array}{c}\text { Strain } \\
\text { No. }\end{array}$} & \multirow[b]{2}{*}{ Serotype } & \multicolumn{8}{|c|}{$\mathrm{MIC}(\mu \mathrm{g} / \mathrm{m} l)$} \\
\hline & & AMP & CHP & CIP & GEN & TET & TRI & $\mathrm{BKC}$ & CHX \\
\hline SA010 & $S$. Anatum & 256 & 32 & 0.5 & 32 & 256 & 256 & 32 & 4 \\
\hline SA034 & $S$. Give & 256 & 32 & 0.125 & 0.5 & 128 & 256 & 128 & 16 \\
\hline SA059 & S. Virchow & 16 & 32 & 1 & 0.25 & 8 & 1 & 256 & 32 \\
\hline SA070 & S. Bsilla & 256 & 64 & 0.03 & 0.5 & 256 & 1 & 128 & 16 \\
\hline SA105 & S. Orion & 4 & 16 & 0.03 & 0.5 & 64 & 1 & 256 & 32 \\
\hline
\end{tabular}

Table 6. Minimum Inhibitory Concentrations (MICs) for BKC and antibiotics in the presence/absence of CCCP of Salmonella isolates

\begin{tabular}{llllllllll}
\hline Strains & CCCP & \multicolumn{8}{c}{ MIC $(\mu \mathrm{g} / \mathrm{m} l)$} \\
\cline { 3 - 9 } & $(\mu \mathrm{M})$ & BKC & AMP & CHP & CIP & ERY & GEN & TET & TRI \\
\hline SA030 & 0 & 4 & 0.5 & 1 & 0.03 & 2 & 0.25 & 0.5 & 0.13 \\
& 50 & 4 & n.d. & 2 & n.d. & 2 & n.d. & n.d. & n.d. \\
SA065 & 0 & 32 & 1 & 64 & 0.03 & 8 & 0.25 & 1 & 0.13 \\
& 50 & 32 & n.d. & 64 & n.d. & 16 & n.d. & n.d. & n.d. \\
\hline
\end{tabular}

n.d., Not determined.

MICs to CHX of $8 \mu \mathrm{g} / \mathrm{m} l$, grew past the first passage and its growth ended at a concentration of $1024 \mu \mathrm{g} / \mathrm{ml}$. All four randomly-picked from the final exposure had MICs for $\mathrm{CHX}$ of $256 \mu \mathrm{g} / \mathrm{m} l$. These isolates were cyclohexane susceptible and their MICs for antibiotics were not different from SA027. The presence of CCCP did not restore their susceptibility to $\mathrm{CHX}$. The high MIC for $\mathrm{CHX}$ was not lost 20 days after passage in CHX-free medium (data not shown).

\section{DISCUSSION}

The results in this study show that multidrug-resistance is common in Salmonella isolates from poultry and swine, which are similar to those previously reported in Thailand $[4,20]$ and from other countries $[7,9,13]$. High proportions of resistant $S$. enterica from swine and poultry were in agreement with a recent study conducted in Thailand that reported the highest resistance rate in Salmonella spp. at the farm [20]. Low levels of ciprofloxacin resistance were observed, which was comparable to other studies of humans and foods [4, 14]. However, the prevalence of ciprofloxacin resistance from this study was slightly higher in comparison to the earlier study in farm sectors, where no resistance to the agent was found [20]. The differences may be associated with differences in laboratory techniques or this may be a reflection of the emergence of ciprofloxacin resistance in Salmonella in Thailand, as observed in other enteropathogenic bacteria [11, 12]. Moreover, the higher prevalence of antibiotic resistance in swine observed may be due to the longer length of the raising period on farms that allows longer exposure to antibiotics.

To date, there is very limited data on the susceptibility to disinfectants in Salmonella in the farm of origin and the most recent epidemiological data was reported by Aarestrup and Hasman [1] in Denmark. In comparison to the study of Danish broiler flocks, distributions of MICs to disinfectants were very close to our study. The Salmonella isolates in the present study had a broader range of MICs to BKC (4-256 $\mu \mathrm{g} / \mathrm{m} l$ ) and slightly lower MICs for $\mathrm{CuSO}_{4}$ (ca. 2-fold difference). This could be also attributed to experimental variations. Since a few deviations from the general distribution in MICs for BKC, CHX and $\mathrm{ZnCl}_{2}$ were revealed and a single MIC value for $\mathrm{CuSO}_{4}$ was observed, it seems that the Salmonella isolates in this study have no or have developed only a limited degree of resistance to disinfectants.

Several Salmonella isolates with high MICs to BKC and $\mathrm{CHX}$ showed resistance to antibiotics but the isolates with multiple antibiotic-resistant phenotypes did not have higher MICs for BKC or CHX than those with non-multiple antibiotic resistance. This dissociation is consistent to a previous study of clinical isolates from human patients [15] and suggests that multidrug efflux systems may not play an important role as the common cross-resistance mechanism [6].

The association between MAR and cyclohexane tolerance has been previously demonstrated [24]. In our study, 3 of 4 cyclohexane-tolerant Salmonella strains (i.e. SA010, SA034 and SA070) have high level resistance to antibiotics and also have low susceptibility to BKC and CHX. SA010 and SA034 carried bla $a_{\mathrm{TEM}}, d f r A 12$ and tet $A$, which were corresponded to their resistance phenotypes (i.e. AMP-CHPTET-TRI). SA070 were multiple resistant to AMP, CHP and TET and harbored $b l a_{\mathrm{TEM}}$ and $t e t A$. The presence of resistance genes was well correlated to the resistance phenotype. Taken together, our results indicate that these strains were MDR. In view of the chloramphenicol MICs, the MICs of 16 to $32 \mu \mathrm{g} / \mathrm{m} l$ suggests that 4 of 5 cyclohexanetolerant strains (i.e. SA010, SA034, SA059 and SA105) could possibly be MAR if the resistance was not due to the resistance genes. Even though none of these strains con- 
tained cat $A$ and $c a t B$ genes, it is possible that they may carry genes encoding chloramphenicol resistance that were not examined in this study.

The contribution of the proton-dependent efflux systems in reduced susceptibility to $\mathrm{BKC}$ and $\mathrm{CHX}$ was assessed using growth inhibition experiments with an energy uncoupler CCCP. The addition of CCCP restores susceptibility to $\mathrm{BKC}$ in only one strain i.e. SA085, suggesting that the efflux systems driven by PMF (including AcrAB) do not play an importance role in reduced susceptibility to the disinfectants in Salmonella isolates from farms. Further studies, currently in progress, are required to elucidate mechanism(s) underlying low susceptibility to BKC in this strain.

For exposure experiments, the antibiotic-susceptible isolates with low MICs to $\mathrm{BKC}$ and $\mathrm{CHX}$ were grown in increasing concentrations of $\mathrm{BKC}$ and $\mathrm{CHX}$. This was to allow cells to adapt to the disinfectants and increase MICs to them. The mechanism (s) responsible for reduced susceptibility to the antimicrobials was tested if it was the PMFdriven efflux system (s) that could promote cross-resistance to antibiotics. Our results showed that successive exposure to gradually increasing concentrations of BKC could yield mutants with the stable reduced susceptibility to the disinfectant as previously described [10]. Antibiotic susceptibility profiles of SA065 demonstrated cross-resistance to chloramphenicol and erythromycin. The possibility that the unidentified mechanism could be the proton-dependent efflux system was excluded since addition of CCCP could not restore the susceptibility. In a previous study, reserpine that blocks efflux pumps driven by either ATP or PMF was used and it was showed that active efflux pump that uses a different type of energy i.e. ATP and is resistant to inhibition by CCCP contributes to the reduced susceptibility to BKC [5]. Because the focus of this study was to test the contribution of efflux pumps driven by PMF in reduced susceptibility to BKC and CHX, we did not pursue the effect of other efflux pump inhibitors any further. However, this cross-resistance may be as a result of nonspecific reduction in cell permeability that does not allow chemically unrelated molecules into the cells [23]. Even though the responsible mechanism is still unidentified, it confirms that exposure to biocide could select for antibiotic resistance. It is sometimes argued that the development of reduced susceptibility to disinfectants in laboratory studies is far from what actually happens in practice because disinfectants at the in-use concentration would kill all bacteria. However, disinfectants are regularly used in farms and diluted to sublethal concentrations downstream of their application. Therefore, bacteria could be exposed to subinhibitory level of disinfectants repeatedly in the same manner that was performed in laboratory.

In light of the results obtained, it can be noted the Salmonella isolates have not developed resistance to $\mathrm{BKC}, \mathrm{CHX}$, copper sulfate and zinc chloride. If they have, it is only to a limited degree. The proton-dependent efflux systems do not play a major role in a reduced susceptibility to $\mathrm{BKC}$ and
CHX in Salmonella from farms, whereas exposure to disinfectants at sublethal concentrations increases selective pressure on bacteria to acquire resistance to antibiotics and disinfectants. These findings warrant further studies to provide a better understanding of the possible link between disinfectant usage and antibiotic resistance in Salmonella.

ACKNOWLEDGEMENT. This research was supported by the Thai Government Research Fund in the fiscal year 2006 to R.C.

\section{REFERENCES}

1. Aarestrup, F. M. and Hasman, H. 2004. Susceptibility of different bacterial species isolated from food animals to copper sulphate, zinc chloride and antimicrobial substances used for disinfection. Vet. Microbiol. 100: 83-89.

2. Asako, H., Nakajima, H., Kobayashi, K., Kobayashi, M. and Aono, R. 1997. Organic solvent tolerance and antibiotic resistance increased by overexpression of marA in Escherichia coli. Appl. Environ. Microbiol. 63: 1428-1433.

3. Boddie, R. L., Nickerson, S. C. and Adkinson, R. W. 1997. Efficacies of teat germicides containing $0.5 \%$ chlorhexidine and 1\% iodine during experimental challenge with Staphylococcus aureus and Streptococcus agalactiae. J. Dairy Sci. 80: 2809-2814.

4. Boonmar, S., Bangtrakulnonth, A., Pornruangwong, S., Samosornsuk, S., Kaneko, K. and Ogawa, M. 1998. Significant increase in antibiotic resistance of Salmonella isolates from human beings and chicken meat in Thailand. Vet. Microbiol. 62: $73-80$.

5. Braoudaki, M. and Hilton, A. C. 2005. Mechanisms of resistance in Salmonella enterica adapted to erythromycin, benzalkonium chloride and triclosan. Int. J. Antimicrob. Agents 25: 31-37.

6. Chuanchuen, R., Khemtong, S. and Padungtod, P. 2007. Occurrence of qacE/qacEAl genes and their correlation with class 1 integrons in Salmonella enterica isolates from poultry and swine. Southeast Asian J. Trop. Med. Public. Health 38: 855-862.

7. Chung, Y. H., Kim, S. Y. and Chang, Y. H. 2003. Prevalence and antibiotic susceptibility of Salmonella isolated from foods in Korea from 1993 to 2001. J. Food Prot. 66: 1154-1157.

8. Dawson, B. and Trapp, R. 2004. Basic and clinical biostatistics, 4 ed. McGraw-Hill, Boston.

9. Gebreyes, W. A. and Thakur, S. 2005. Multidrug-resistant Salmonella enterica serovar Muenchen from pigs and humans and potential interserovar transfer of antimicrobial resistance. Antimicrob. Agents Chemother. 49: 503-511.

10. Gradel, K. O., Randall, L., Sayers, A. R. and Davies, R. H. 2005. Possible associations between Salmonella persistence in poultry houses and resistance to commonly used disinfectants and a putative role of mar. Vet. Microbiol. 107: 127-138.

11. Hanson, R., Kaneene, J. B., Padungtod, P. Hirokawa, K. and Zeno, C. 2002. Prevalence of Salmonella and E. coli, and their resistance to antimicrobial agents, in farming communities in northern Thailand. Southeast Asian J. Trop. Med. Public Health 33 (Suppl 3): 120-126.

12. Hoge, C. W., Gambel, J. M., Srijan, A., Pitarangsi, C. and Echeverria, P. 1998. Trends in antibiotic resistance among diarrheal pathogens isolated in Thailand over 15 years. Clin. Infect. Dis. 26: 341-345. 
13. Hsu, S. C., Chiu, T. H., Pang, J. C., Hsuan-Yuan, C. H., Chang, G. N. and Tsen, H. Y. 2006. Characterisation of antimicrobial resistance patterns and class 1 integrons among Escherichia coli and Salmonella enterica serovar Choleraesuis strains isolated from humans and swine in Taiwan. Int. J. Antimicrob. Agents 27: 383-391.

14. Isenbarger, D. W., Hoge, C. W., Srijan, A., Pitarangsi, C., Vithayasai, N., Bodhidatta, L., Hickey, K. W. and Cam, P. D. 2002. Comparative antibiotic resistance of diarrheal pathogens from Vietnam and Thailand, 1996-1999. Emerg. Infect. Dis. 8: $175-180$

15. Kucken, D., Feucht, H. and Kaulfers, P. 2000. Association of $q a c E$ and $q a c E$ delta 1 with multiple resistance to antibiotics and antiseptics in clinical isolates of Gram-negative bacteria. FEMS Microbiol. Lett. 183: 95-98.

16. Lacroix, F. J., Cloeckaert, A., Grepinet, O., Pinault, C., Popoff, M. Y., Waxin, H. and Pardon, P. 1996. Salmonella typhimurium acrB-like gene: identification and role in resistance to biliary salts and detergents and in murine infection. FEMS Microbiol. Lett. 135: 161-167.

17. Leverstein-van Hall, M. A., Box, A. T., Blok, H. E., Paauw, A., Fluit, A. C. and Verhoef, J. 2002. Evidence of extensive interspecies transfer of integron-mediated antimicrobial resistance genes among multidrug-resistant Enterobacteriaceae in a clinical setting. J. Infect. Dis. 186: 49-56.

18. McDonnell, G. and Russell, A. D. 1999. Antiseptics and disinfectants: activity, action, and resistance. Clin. Microbiol. Rev. 12: $147-179$.

19. NCCLS. 2002. Performance Standards for Antimicrobial Disk and Dilution Susceptibility Tests for Bacteria Isolated from Animals; Approved Standard-Second Edition. NCCLS docu- ment M31-A2. NCCLS, PA, U.S.A.

20. Padungtod, P. and Kaneene, J. B. 2006. Salmonella in food animals and humans in northern Thailand. Int. J. Food Microbiol. 108: $346-354$.

21. Poole, K. 2002. Mechanisms of bacterial biocide and antibiotic resistance. J. Appl. Microbiol. 92: 55S-64S.

22. Randall, L. P., Cooles, S. W., Sayers, A. R. and Woodward, M. J. 2001. Association between cyclohexane resistance in Salmonella of different serovars and increased resistance to multiple antibiotics, disinfectants and dyes. J. Med. Microbiol. 50: 919924.

23. Tattawasart, U., Maillard, J. Y., Furr, J. R. and Russell, A. D. 1999. Development of resistance to chlorhexidine diacetate and cetylpyridinium chloride in Pseudomonas stutzeri and changes in antibiotic susceptibility. J. Hosp. Infect. 42: 219229.

24. Webber, M., Buckley, A. M., Randall, L. P., Woodward, M. J. and Piddock, L. J. 2006. Overexpression of marA, soxS and $a c r B$ in veterinary isolates of Salmonella enterica rarely correlates with cyclohexane tolerance. J. Antimicrob. Chemother. 57: 673-679.

25. White, D. G., Goldman, J. D., Demple, B. and Levy, S. B. 1997. Role of the acr $A B$ locus in organic solvent tolerance mediated by expression of marA, soxS, or robA in Escherichia coli. J. Bacteriol. 179: 6122-6126.

26. Woods, C. R., Versalovic, J., Koeuth, T. and Lupski, J. R. 1993. Whole-cell repetitive element sequence-based polymerase chain reaction allows rapid assessment of clonal relationships of bacterial isolates. J. Clin. Microbiol. 31: $1927-$ 1931. 